\title{
Nefropatía terminal en pacientes de un hospital de referencia en El Salvador
}

\author{
Ramón García Trabanino, ${ }^{1}$ Raúl Aguilar, ${ }^{2}$ Carlos Reyes Silva, ${ }^{1}$ \\ Manuel Ortiz Mercado ${ }^{1}$ y Ricardo Leiva Merino ${ }^{3}$
}

RESUMEN Objetivos. El Salvador es un país con alta mortalidad por nefropatía terminal (NT). El objetivo de este estudio consistió en conocer las características epidemiológicas de una serie de nuevos casos de NT atendidos en un hospital de referencia de este país.

Métodos. Se realizó un estudio transversal de todos los nuevos casos que iniciaron diálisis crónica entre noviembre de 1999 y marzo de 2000. Mediante una entrevista personal se obtuvieron datos sobre aspectos clínicos, demográficos, laborales y ambientales.

Resultados. Durante los 5 meses que duró el estudio se observaron 205 nuevos casos de NT. Entre los 202 entrevistados, se diferenciaron claramente dos grupos: uno de 67 pacientes (33\%) con factores de riesgo conocidos de NT, similares a los de países desarrollados (fundamentalmente, diabetes mellitus, hipertensión arterial y consumo crónico de antiinflamatorios no esteroideos), y otro de 135 pacientes (67\%) con características peculiares, en los que no se pudo detectar ningún factor asociado. La mayoría de estos últimos pacientes eran hombres, agricultores, habitantes de zonas costeras o adyacentes a ríos, que años atrás habian estado expuestos, sin protección, a insecticidas o plaguicidas agrícolas por razones laborales.

Conclusiones. Se ha identificado un importante grupo de pacientes con NT que aparentemente carecen de una causa para su enfermedad y que presentan características peculiares que permiten sospechar una relación con la exposición laboral a insecticidas o plaguicidas. Son necesarios nuevos estudios para confirmar esta hipótesis.

Palabras clave Nefropatía terminal, características epidemiológicas, insecticidas, plaguicidas.

En las dos últimas décadas ha habido un aumento de las nefropatías terminales (NT) en todo el mundo (1). En países pobres esto plantea problemas asistenciales graves (2), y más aún en un país como El Salvador, cuyas tasas de morbilidad y mortalidad son de las

\footnotetext{
Departamento de Medicina Interna, Hospital Nacional Rosales, San Salvador, El Salvador. Toda la correspondencia debe ser enviada a Ramón García Trabanino, Fundación Puigvert, IUNA, Calle Cartagena 340, 08025, Barcelona, España.

2 Unidad de Investigaciones Médicas, Hospital Nacional Rosales, San Salvador, El Salvador.

3 Servicio de Nefrología, Hospital Nacional Rosales, San Salvador, El Salvador.
}

más altas de América Latina (3). Si bien el sistema de salud de El Salvador se encuentra en un proceso de reforma y se espera que cambie en el mediano plazo, hasta la fecha aún posee tres sectores. El primero es el de los servicios médicos privados (clínicas y hospitales), utilizado por un pequeño porcentaje de la población (aproximadamente $5 \%$ ) con ingresos suficientes para pagarlos o que están adscritos a seguros privados. El segundo es el Instituto del Seguro Social, que cubre a cerca de $25 \%$ de los habitantes y es financiado por una mezcla de fondos provenientes del gobierno, de los empleadores y de los trabajadores. Ambos efectúan trasplantes renales para adultos. $\mathrm{El} 70 \%$ restante de la población, desempleada o con salarios muy bajos para obtener un seguro, está cubierta por el Sistema Nacional de Salud Pública, administrado por el Ministerio de Salud. El Hospital Nacional Rosales, con 450 camas, es el mayor y más antiguo de su clase y pertenece a esta última red. Es el centro más importante de tercer nivel para adultos que requieren atención en las subespecialidades médicas y quirúrgicas, y posee, además, el principal Servicio de Nefrología del sistema público, dotado a la fecha con 3 nefrólogos, 10 
enfermeras especializadas y 8 máquinas de hemodiálisis.

Estadísticas locales han demostrado que la NT es el diagnóstico de egreso más frecuente de todo el hospital, registrándose unos 300 a 400 pacientes nuevos por año. Debido a que existe un número limitado de máquinas de hemodiálisis y a que no es posible financiar un programa de trasplantes, casi todos los pacientes son admitidos a un programa de diálisis peritoneal intermitente con catéter peritoneal rígido. En este estudio se investigan las características epidemiológicas básicas de una serie de nuevos casos de NT, con el propósito de encontrar posibles medidas de prevención y detección precoz.

\section{MATERIALES Y MÉTODOS}

Se definió como nuevo caso de NT a todo paciente con insuficiencia renal crónica (IRC) que necesitara iniciar tratamiento crónico con cualquier tipo de diálisis (1). Como causa de la NT se consideró la enfermedad subyacente o el diagnóstico primario de los pacientes (1). Una vez obtenido el consentimiento informado de los pacientes, se llevó a cabo una entrevista personal en la cual se utilizó un cuestionario para recolectar información acerca del sexo, edad, procedencia, ocupación, consumo de alcohol y medicamentos, recetados o no, exposición laboral a insecticidas o plaguicidas (sin especificar nombres) usados en la agricultura, antecedentes personales médicos y quirúrgicos, antecedentes familiares y enfermedades concomitantes. Los pacientes elegibles fueron todos aquellos con IRC que ingresaron en el hospital para su primera hemodiálisis o diálisis peritoneal entre el 1 de noviembre de 1999 y el 30 de marzo de 2000. Se excluyeron los que rehusaron contestar las preguntas o no pudieron hacerlo por estar demasiado enfermos para conversar o por padecer trastornos mentales.

\section{RESULTADOS}

Durante los 5 meses que duró el estudio se atendieron 205 nuevos pacien-

CUADRO 1. Diagnósticos primarios de 202 nuevos casos de nefropatía terminal en un hospital de referencia. El Salvador, noviembre de 1999 a marzo de 2000

\begin{tabular}{lrrr}
\hline \multicolumn{1}{c}{ Diagnóstico primario } & & \multicolumn{2}{c}{$\%$} \\
\cline { 3 - 4 } & No. & Parcial & Total \\
\hline Diabetes mellitus & $30^{a}$ & 44,8 & 14,9 \\
Hipertensión arterial & 15 & 22,4 & 7,4 \\
Consumo diario de AINE o Artritex & 9 & 13,4 & 4,5 \\
Enfermedades quísticas renales & 4 & 6,0 & 2,0 \\
Litiasis renalc & 3 & 4,5 & 1,5 \\
Glomerulonefritis & 1 & 1,5 & 0,5 \\
Síndrome nefrótico & 1 & 1,5 & 0,5 \\
Mieloma múltiple & 1 & 1,5 & 0,5 \\
Infecciones renales de repetición & 1 & 1,5 & 0,5 \\
Uropatía obstructiva crónica & 1 & 1,5 & 0,5 \\
Hemorragia masiva & 1 & 1,5 & 0,5 \\
$\quad$ Subtotal de casos con diagnóstico primario & 67 & 100 & 33,2 \\
Casos sin diagnóstico primario & 135 & & 66,8 \\
$\quad$ Total & 202 & & 100 \\
\hline
\end{tabular}

a De tipo 2 en 29 pacientes y de tipo 1 en el otro.

b Medicamento compuesto por dexametasona, 0,5 mg; N-acetil-p-aminofenol, $125 \mathrm{mg}$, y dipirona, $250 \mathrm{mg}$. De estos nueve pacientes, siete tomaban ambos, uno solo AINE y otro solo Artritex. Los AINE más frecuentemente mencionados fueron: ibuprofeno, piroxicam e indometacina. AINE = antiinflamatorios no esteroideos.

c Litiasis renal más nefrectomía en dos pacientes y litiasis renal más pielonefritis en uno.

tes con NT, de los cuales solo 3 no quisieron o no pudieron ser interrogados. Sesenta y siete de los 202 pacientes (33,2\%; grupo I) presentaban un diagnóstico primario o factor etiológico claramente reconocido como causa de NT; los tres más comunes fueron la diabetes mellitus, la hipertensión arterial y la ingesta crónica de antiinflamatorios no esteroideos (AINE). Sin embargo, los otros 135 casos $(66,8 \%$; grupo II) no presentaban enfermedades previas ni factores de riesgo conocidos que explicaran su insuficiencia renal (cuadro 1). Los perfiles epidemiológicos de estos dos grupos de pacientes fueron muy diferentes.

El grupo I presentó una media ( \pm desviación estándar) de $56( \pm 15,7)$ años de edad, y el número de hombres y mujeres (32 y 35, respectivamente) fue similar (cuadro 2). En 16 mujeres y 14 hombres se había diagnosticado diabetes (cuadro 1), de tipo 2 en 29 de ellos. Estos pacientes procedían principalmente de áreas urbanas y cerca de la mitad de ellos trabajaban como pequeños comerciantes o amas de casa; solamente $20,8 \%$ se ocupaba en labores agrícolas (cuadro 3). El 25,4\% dijo haber tenido algún tipo de contacto con herbicidas o plaguicidas (cuadro
4). Además, se observó una alta frecuencia de antecedentes médicos (94\%), quirúrgicos $(53,7 \%)$ y farmacológicos $(88,1 \%)$ en los pacientes de este grupo (cuadro 4).

El grupo II presentó características peculiares, diferentes de las del grupo anterior, con predominio del sexo masculino (118 hombres frente a 17 mujeres) y con una edad media más joven, de $51( \pm 16,1)$ años (cuadro 2). La gran mayoría de ellos eran o habían sido agricultores (63\% como trabajo actual, y $75 \%$ considerando ocupaciones previas) (cuadro 3), y casi todos procedían de zonas rurales, particularmente de zonas donde hace una o dos décadas hubo plantaciones y áreas de cultivo de café, maíz y, sobre todo, algodón. El $73,3 \%$ recordaba haber tenido algún tipo de contacto frecuente con herbicidas o plaguicidas, habitualmente sin protección adecuada (guantes, máscara o ropa especial) (cuadro 4). Estos pacientes referían haber gozado de buena salud hasta que adquirieron la IRC y llegaron a la NT, pues muy pocos de ellos presentaban antecedentes médicos $(4,4 \%)$, quirúrgicos $(17 \%)$ o farmacológicos $(6,7 \%)$ (cuadro 4 ).

De los 202 pacientes encuestados, $24 \%$ dijo consumir agua de pozos, 
CUADRO 2. Distribución por edad y sexo de 202 nuevos casos de nefropatía terminal en un hospital de referencia. El Salvador, noviembre de 1999 a marzo de 2000

\begin{tabular}{|c|c|c|c|c|c|c|}
\hline & \multicolumn{2}{|c|}{$\begin{array}{l}\text { Grupo } \mathrm{I}^{\mathrm{a}} \\
(n=67)\end{array}$} & \multicolumn{2}{|c|}{$\begin{array}{l}\text { Grupo II } \\
(n=135)\end{array}$} & \multicolumn{2}{|c|}{ Total } \\
\hline & No. & $\%$ & No. & $\%$ & No. & $\%$ \\
\hline \multicolumn{7}{|l|}{ Edad $^{c}$} \\
\hline$\leq 19$ & 2 & 3,0 & 3 & 2,2 & 5 & 2,5 \\
\hline 20 a 29 & 4 & 6,0 & 12 & 8,9 & 16 & 7,9 \\
\hline 30 a 39 & 4 & 6,0 & 12 & 8,9 & 16 & 7,9 \\
\hline 40 a 49 & 9 & 13,4 & 27 & 20,0 & 36 & 17,8 \\
\hline 50 a 59 & 14 & 20,9 & 41 & 30,4 & 55 & 27,2 \\
\hline 60 a 69 & 23 & 34,3 & 18 & 13,3 & 41 & 20,3 \\
\hline$\geq 70$ & 11 & 16,4 & 22 & 16,3 & 33 & 16,3 \\
\hline Total & 67 & 100 & 135 & 100 & 202 & 100 \\
\hline \multicolumn{7}{|l|}{ Sexo } \\
\hline Masculino & 32 & 47,8 & 118 & 87,4 & 150 & 74,3 \\
\hline Femenino & 35 & 52,2 & 17 & 12,6 & 52 & 25,7 \\
\hline Total & 67 & 100 & 135 & 100 & 202 & 100 \\
\hline
\end{tabular}

CUADRO 3. Ocupación u oficio de 202 nuevos casos de nefropatía terminal en un hospital de referencia. El Salvador, noviembre de 1999 a marzo de 2000

\begin{tabular}{|c|c|c|c|c|c|c|}
\hline \multirow[b]{2}{*}{ Ocupación } & \multicolumn{2}{|c|}{$\begin{array}{l}\text { Grupo }\left.\right|^{\mathrm{a}} \\
(n=67)\end{array}$} & \multicolumn{2}{|c|}{$\begin{array}{l}\text { Grupo IIb } \\
(n=135)\end{array}$} & \multicolumn{2}{|c|}{ Total } \\
\hline & No. & $\%$ & No. & $\%$ & No. & $\%$ \\
\hline Agricultor & 14 & 20,8 & 85 & 63,0 & 99 & 49,0 \\
\hline Domésticos & 15 & 22,4 & 10 & 7,4 & 25 & 12,3 \\
\hline No trabaja & 10 & 14,9 & 5 & 3,7 & 15 & 7,4 \\
\hline Comerciante & 8 & 11,9 & 2 & 1,5 & 10 & 4,9 \\
\hline Cocinera & 4 & 6,0 & 1 & 0,7 & 5 & 2,5 \\
\hline Motorista & 1 & 1,5 & 4 & 3,0 & 5 & 2,5 \\
\hline Albañil & - & - & 5 & 3,7 & 5 & 2,5 \\
\hline Mecánico & 3 & 4,5 & 1 & 0,7 & 4 & 2,0 \\
\hline Costurera & 2 & 3,0 & 2 & 1,5 & 4 & 2,0 \\
\hline Estudiante & 2 & 3,0 & 2 & 1,5 & 4 & 2,0 \\
\hline Obrero industria química ${ }^{\mathrm{C}}$ & - & - & 2 & 1,5 & 2 & 1,0 \\
\hline Zapatero & 1 & 1,5 & 1 & 0,7 & 2 & 1,0 \\
\hline Panadero & - & - & 2 & 1,5 & 2 & 1,0 \\
\hline Carpintero & 1 & 1,5 & 1 & 0,7 & 2 & 1,0 \\
\hline Otros & 6 & 9,0 & 12 & 8,9 & 18 & 8,9 \\
\hline Total & 67 & 100 & 135 & 100 & 202 & 100 \\
\hline
\end{tabular}

aproximadamente la mitad admitió ser fumador o haber fumado con regularidad en el pasado, y $63 \%$ reconoció haber tenido un consumo importante de bebidas alcohólicas durante los años anteriores. En estos aspectos no se observaron diferencias notorias entre los dos grupos.

\section{DISCUSIÓN}

Resulta llamativo recibir a 205 enfermos nuevos para diálisis en un mismo centro en tan solo 5 meses. Por otra parte, las diferencias entre los dos grupos fueron realmente sorprendentes. El primero tenía casi todos los factores de riesgo conocidos de NT, aunque eran un poco más jóvenes que en otros países y regiones, como Estados Unidos (1), Europa $(4,5)$ y Kuwait (6), donde la NT suele aparecer alrededor de la séptima década de la vida. No detectamos predominancia de uno $\mathrm{u}$ otro sexo, lo cual también sucede en naciones ricas (1) y pobres (7). Tampoco fue sorpresa encontrar sujetos con factores de riesgo conocidos, como la diabetes, dado que ocurre en Estados Unidos (8), Canadá (9), Europa (10) y Oriente Medio (6); lo mismo la hipertensión arterial (11), que ocupa el segundo puesto en Estados Unidos (1) y va en aumento en Europa (4). La ingesta de analgésicos, que no se cuantificó en este estudio, es otro factor de riesgo bien conocido (12). En cambio, no se detectaron factores de riesgo conocidos en el segundo grupo de pacientes, que constituyeron dos tercios de la muestra total. Casi todos eran hombres que vivían en las zonas bajas del país, cercanas a la costa, por las que fluye un río importante.

Posiblemente la principal causa de la NT en los pacientes del primer grupo haya sido que la diabetes, la hipertensión arterial y la ingesta crónica de AINE ocurrieran bajo escasa o ninguna supervisión de profesionales, por causa de la pobreza, el analfabetismo y el difícil acceso a cuidados periódicos de salud, que previenen o retardan la aparición de la NT (18). Sin embargo, hemos identificado una importante población de pacientes masculinos con NT procedentes de zonas de cultivo costeras o adyacentes a ríos, en su mayoría agricultores, y que recordaban haber tenido contacto laboral frecuente y sin protección adecuada con insecticidas o plaguicidas agrícolas. Según datos de la Universidad Nacional y del Ministerio de Agricultura, entre los más usados hace dos décadas estaban los carbamatos, el 
CUADRO 4. Antecedentes de 202 nuevos casos de nefropatía terminal en un hospital de referencia. El Salvador, noviembre de 1999 a marzo de 2000

\begin{tabular}{|c|c|c|c|c|c|c|}
\hline & \multicolumn{2}{|c|}{$\begin{array}{l}\text { Grupo Ia } \\
(n=67)\end{array}$} & \multicolumn{2}{|c|}{$\begin{array}{l}\text { Grupo II } \\
(n=135)\end{array}$} & \multicolumn{2}{|c|}{ Total } \\
\hline & No. & $\%$ & No. & $\%$ & No. & $\%$ \\
\hline \multicolumn{7}{|c|}{$\begin{array}{l}\text { Antecedentes de contacto con } \\
\text { herbicidas y plaguicidas }\end{array}$} \\
\hline Sí & 17 & 25,4 & 99 & 73,3 & 116 & 57,4 \\
\hline No & 50 & 74,6 & 36 & 26,6 & 86 & 42,6 \\
\hline \multicolumn{7}{|c|}{ Antecedentes médicos } \\
\hline Sí & 63 & 94,0 & 6 & 4,4 & 69 & 34,2 \\
\hline No & 4 & 6,0 & 129 & 95,6 & 133 & 65,8 \\
\hline \multicolumn{7}{|c|}{ Antecedentes farmacológicos } \\
\hline Sí & 59 & 88,1 & 9 & 6,7 & 68 & 33,7 \\
\hline No & 8 & 11,9 & 126 & 93,3 & 134 & 66,3 \\
\hline \multicolumn{7}{|c|}{ Antecedentes quirúrgicos } \\
\hline Sí & 36 & 53,7 & 23 & 17,0 & 59 & 29,2 \\
\hline No & 31 & 46,3 & 112 & 83,0 & 143 & 70,8 \\
\hline
\end{tabular}

a Pacientes con diagnóstico primario o factores de riesgo conocidos de nefropatía terminal.

b Pacientes sin diagnóstico primario ni factores de riesgo conocidos de nefropatía terminal.

Nota: $52,2 \%$ de los pacientes del grupo I tienen algún antecedente familiar relevante (hipertensión arterial, diabetes u otro).

$26,7 \%$ de los pacientes del grupo II tienen algún antecedente familiar relevante, pero ninguno de ellos relacionado con nefropatías hereditarias o familiares conocidas.

DDT, los organofosforados y organoclorados y los piretroides. En este sentido, es posible que algún tóxico presente en los insecticidas pueda haber causado daño renal subclínico, pero progresivo, cuya manifestación clínica pudo haberse retardado en más de una década debido a la dieta hipopro- teínica que consumen las personas pobres de Centroamérica, un factor bien conocido que retrasa la aparición de la NT (19). También es probable que, debido a la contaminación de los mantos acuíferos, haya en el agua metales pesados nefrotóxicos (20), aunque eso no explicaría el hecho de que la mayoría de los pacientes fueran del sexo masculino y agricultores.

Un estudio descriptivo como el presente no pretende demostrar nada y más bien intenta llamar la atención sobre el grave problema que representan para un país subdesarrollado los pacientes con IRC que llegan hasta la NT y que dependen de la diálisis o de alguna otra forma de reemplazo de la función renal en una sociedad que no puede proporcionarles cuidados apropiados por la carencia de recursos financieros. Para dilucidar el verdadero significado de estos casos de NT sin factores de riesgo conocidos serán necesarios nuevos estudios.

Agradecimientos: A los doctores Angel Díaz y Zulma Cruz por haber revisado el manuscrito y por sus valiosas sugerencias.

\section{REFERENCIAS}

1. United States Renal Data System. Incidence and Prevalence of end-stage renal disease. 1999 Annual Data Report. Bethesda, MD: National Institutes of Health/NIDDKD; 1999. Pp:25-38. Disponible en http://www.usrds. org/adr_1999.htm (acceso el 29 julio 2002).

2. Plata R, Silva C, Yahuita J, Perez L, Schieppati A, Remuzzi G. The first clinical and epidemiological programme on renal disease in Bolivia: a model for prevention and early diagnosis of renal diseases in the developing countries. Nephrol Dial Transplant 1998;13: 3034-3036.

3. Mazzuchi M, Schwedt E, Fernandez JM, Cusumano AM, Ancao MS, Poblete $\mathrm{H}$, et al. Latin American Registry of dialysis and renal transplantation: 1993 annual dialysis data report. Nephrol Dial Transplant 1997;12:25212527.

4. Rutkowski B. Changing pattern of end-stage renal disease in central and eastern Europe. Nephrol Dial Transplant 2000;15:156-160.

5. Jungers $P$, Chauveau $P$, Descamps-Latscha B, Labrunie M, Giraud E, Man NK, et al. Age and gender-related incidence of chronic renal plant 1994;9:532-538. failure in a French urban area: a prospective epidemiologic study. Nephrol Dial Transplant 1996;11:1542-1546.

6. El-Reshaid K, Johny KV, Sugathan TN, Hakim A, Georgous M, Nampoory MR. Endstage renal disease and renal replacement therapy in Kuwait - epidemiological profile over the past $4 \frac{1}{2}$ years. Nephrol Dial Trans-

7. Khan IH, Thereska N, Barbullushi M, MacLeod AM. The epidemiology of chronic renal failure and provision of renal services in Albania. Nephrol Dial Transplant 1996;11: 1751-1754.

8. Centers for Disease Control. End-stage renal disease associated with diabetes - United States 1988. MMWR 1989;38:546-548.

9. Pylypchuk G, Beaubien E. Diabetic nephropathy: prevention and early referral. Can Fam Physician 2000;46:636-642.

10. Ibrahim HA, Vora JP. Diabetic nephropathy. Bailliere's Best Pract Res Clin Endocrinol Metab 1999;13:239-264.

11. Iseki K, Ikemiya Y, Fukiyama K. Blood pressure and risk of end-stage renal disease in a screened cohort. Kidney Int 1996;55(Supl) S69-S71.

12. Perneger TV, Whelton PK, Klag MJ. Risk of kidney failure associated with the use of acetaminophen, aspirin, and nonsteroidal antiinflammatory drugs. N Eng J Med 1994;331: 1675-1679.

13. Orth SR, Stöckmann A, Conradt C, Ritz E, Ferro M, Kreusser W, et al. Smoking as a risk factor for end-stage renal failure in men with primary renal disease. Kidney Int 1998;54: 926-931.

14. Regalado M, Yang S, Wesson DE. Cigarette smoking is associated with augmented progression of renal insufficiency in severe essential hypertension. Am J Kidney Dis 2000;35: 687-694.

15. Perneger TV, Whelton PK, Puddey IB, Klag MJ. Risk of end-stage renal disease with alcohol consumption. Am J Epidemiol 1999;150: 1275-1281.

16. Kasiske BL, Rith-Najarian S, Casper ML, Croft JB. American Indian heritage and risk factors for renal injury. Kidney Int 1998;54:13051310 
17. Strojek K, Grzeszczak W, Morawin E, Adamski M, Lacka B, Rudzki H, et al. Nephropathy of type II diabetes: evidence for hereditary factors? Kidney Int 1997;51:1602-1607.

18. Stefanski A, Amann K, Ritz E. To prevent progression: ACE inhibitors, calcium antagonists or both? Nephrol Dial Transplant 1995;10: 151-153.
19. Gretz N, Giovannetti S, Strauch M. Low protein diet and progression of chronic renal failure. Klin Wochenschr 1988;66:416-417.

20. Sánchez-Fructuoso AI, Torralbo A, Arroyo M, Luque M, Ruilope LM, Santos JL, et al. Occult lead intoxication as a cause of hypertension and renal failure. Nephrol Dial Transplant 1996;11: 1775-1780.
Manuscrito recibido el 12 de marzo de 2002. Aceptado para publicación el 21 de junio de 2002

ABSTRACT Objective. El Salvador is a country with high mortality from end-stage renal disease (ESRD). The objective of this study was to determine the epidemiological characteristics of a series of new cases of ESRD seen in a referral hospital in the country.

Methods. A cross-sectional study was conducted of all the new cases that initiated chronic dialysis between November 1999 and March 2000. Using a personal interview, data were obtained on the patients' clinical, demographic, and occupational characteristics, among others.

Results. During the five months that the study lasted, 205 new cases of ESRD were observed. Among the 202 interviewees, two groups were clearly distinguished. One group, of 67 patients (33\%), had known risk factors for ESRD, similar to those for developed countries (basically, diabetes mellitus, hypertension, and chronic consumption of non-steroidal anti-inflammatories). Another group of 135 patients $(67 \%)$ had unusual characteristics that were not associated with the known risk factors. The majority of the patients in this second group were male, farmers, residents of coastal areas or areas next to rivers, and some years before had been exposed, without adequate protection, to agricultural insecticides or pesticides through their work.

Conclusions. We have identified an important group of patients with ESRD who seem to lack a cause for their disease. Their special characteristics make it possible to suspect a relationship with the occupational exposure to insecticides or pesticides. New studies are needed in order to confirm this hypothesis.

\section{Coloquio Internacional sobre la Infección Congénita por Trypanosoma cruzi}

Fechas: 6 a 8 de noviembre de 2002

Lugar: Cochabamba, Bolivia

Tema: $\quad$ Infección congénita por Trypanososma cruzi: de los mecanismos de transmisión a una estrategia de diagnóstico y control.

Este Coloquio Internacional será organizado por la Universidad Mayor de San Simón (UMSS, Prof. F. Torrico) y la Universidad Libre de Bruselas (ULB, Prof Y. Carlier), con el apoyo de la Cooperación Belga (CIUF/CUD/CGRI/APEFE).

Información:

Correo electrónico: ftorrico@med.umss.edu.bo y carlier@ulb.ac.be 